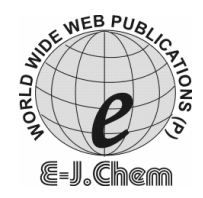

\title{
Synthesis and Antimicrobial Studies of Some Bis-Glycosyl Isodithiobiurets
}

\author{
PRASHANT R. MAHALLE and SHIRISH P. DESHMUKH* \\ P. G. Department of Chemistry, \\ Shri Shivaji College, Akola - 444001 (M.S.), India. \\ prmahalle@ rediffmail.com
}

Received 22 October 2009; Accepted 15 December 2009

\begin{abstract}
Certain 2-S-tetra- $O$-benzoyl- $D$-glucopyranosyl-1-aryl-5-tetra- $O$ acetyl $-\beta$ - $D$ - galactopyranosyl-2, 4 - isodithiobiurets have been synthesized by the interaction of tetra- $O$-acetyl- $\beta$ - $D$-galactopyranosyl isothiocyante and various $S$ - tetra- $O$-benzoyl- $D$ - glucopyranosyl-1-aryl isothiocarbamides. The newly synthesized compounds were screened for their antimicrobial activities.
\end{abstract}

Keywords: Synthesis, Antimicrobial studies, Bis-glycosyl isodithiobiurets

\section{Introduction}

Aryl/alkyl isothiocarbamides, due to their basic nature are found to interact with isothiocyante to form corresponding isodithiobiurests. Several non-glycosidic isodithiobiurests are known for their anticonvulsant and hypnotic activities ${ }^{1}$, several $S$-glucosides have been described in the literature to possess antimicrobial activities ${ }^{2}$. Glycopyranosyl isothiocyanates are attractive synthons in organic chemistry due to their availability and their tendency to undergo nucleophilic additions and cycloadditions. Several $\mathrm{N}$-glycosides have been found several applications in industries as carbohydrate based detergents ${ }^{3}$ and in medicine as antitumour ${ }^{4}$ and antitubercular ${ }^{5}$ activities.

In view of applications of all the compounds described above and our interest in the synthesis of bis glycosyl isodithiobiurets, we report the synthesis of 2-S-tetra- $O$-benzoyl- $D$ glucopyranosyl-1-aryl-5- tetra- $O$-acetyl- $\beta$ - $D$-galactopyranosyl-2, 4 -isodithiobiurets by the interaction of tetra- $O$ - acetyl- $\beta$ - $D$ - galactopyranosyl isothiocyante and various $S$ - tetra- $O$ benzoyl - $D$ - glucopyranosyl - 1-aryl isothiocarbamides.

\section{Experimental}

Melting points are uncorrected. Optical rotations $[\alpha]_{D}$ were measured on a Equip-Tronics digital polarimeter model no. EQ 800 in $\mathrm{CHCl}_{3}$ at $39{ }^{\circ} \mathrm{C}$. IR spectra were recorded on a Perkin-Elmer spectrum RXI $\left(4000-450 \mathrm{~cm}^{-1}\right)$ FTIR spectrometer. ${ }^{1} \mathrm{HNMR}$ were obtained on 
a Bruker DRX-300 (300 MHz FT NMR) NMR spectrometer for a sample in $\mathrm{CDCl}_{3}$ solution with TMS as an internal reference. The mass spectra were recorded on a Joel - SX 102 FAB Mass spectrometer.

Tetra-O-acetyl- $\beta$-D - galactopyranosyl isothiocyanate (1)

Tetra- $O$-acetyl- $\beta$ - galactopyranosyl isothiocyanate (2) was prepared from tetra- $O$-acetyl- $\alpha$ - galactopyranosyl bromide using lead thiocyanate in xylene medium ${ }^{10}$.

$S$ - tetra-O - benzoyl -D - glucopyranosyl 1- aryl isothiocarbamides (2a-g)

$S$-Tetra- $O$ - benzoyl - $D$ - glucopyranosyl 1- aryl isothiocarbamides have been prepared by the methods described earlier which involves interaction of tetra- $O$-benzoyl- $D$-glucopyranosyl bromide with various aryl isothiocarbamides ${ }^{11}$.

2-S-tetra-O-benzoyl-D-glucopyranosyl-5-tetra-O-acetyl- $\beta$-D -galactopyranosyl - 1 - aryl - 2,4 isodithiobiurets (3a- $\mathbf{g})$

$2-S$-tetra $-O$ - benzoyl $-D$ - glucopyranosyl-5-tetra- $O$ - acetyl- $\beta$ - $D$-galactopyranosyl-1- aryl 2 , 4 isodithiobiurets $(\mathbf{3 a}-\mathbf{g})$ were prepared by the condensation of $S$ - tetra- $O$-benzoyl- $D$ glucopyranosyl 1-aryl isothiocarbamides $(\mathbf{2 a - g}, 0.005 \mathrm{M})$ and tetra- $O$-acetyl $\beta-D$ galactopyranosyl isothiocyanate $(1,0.005 \mathrm{M})$ in benzene for $4 \mathrm{~h}$. After the reaction was completed the solvent was distilled off and the sticky residue was triturated with petroleum ether to give the solids. The characterization data of the synthesized products is given in the Table 1.

Table 1. Characterization data of the synthesized products (3a-g).

Reagent: Tetra - $O$ - acetyl- $\beta$ - $D$-galactopyranosyl isothiocyanate; $S$ - tetra $-O$ - benzoyl - $D$ glucopyranosyl - 1 - aryl - isothiocarbamides ( 2a-g).

\begin{tabular}{|c|c|c|c|c|c|c|c|}
\hline \multirow[b]{2}{*}{ S. No. } & \multirow[b]{2}{*}{ Product } & \multirow{2}{*}{$\begin{array}{c}\% \\
\text { Yield }\end{array}$} & \multirow{2}{*}{${ }^{\mathrm{m}} \cdot \mathrm{p}}$. & \multirow{2}{*}{$\begin{array}{c}{[\alpha]_{\mathrm{D}}^{39}} \\
\left(\mathrm{CHCl}_{3}\right)\end{array}$} & \multicolumn{2}{|c|}{ Analysis } & \multirow{2}{*}{$\begin{array}{c}\mathrm{R}_{\mathrm{f}} \\
\left(\mathrm{CCl}_{4}: \mathrm{EtOAc}\right)\end{array}$} \\
\hline & & & & & $\begin{array}{c}\text { Found } \\
\%\end{array}$ & $\begin{array}{c}\text { Required } \\
\%\end{array}$ & \\
\hline \multirow{2}{*}{1} & \multirow{2}{*}{$\mathbf{3 a}$} & \multirow{2}{*}{55} & \multirow{2}{*}{115} & $75.25^{\circ}$ & $\mathrm{N}, 3.62$ & N, 3.62 & 0.77 \\
\hline & & & & $(\mathrm{c}, 0.94)$ & $\mathrm{S}, 5.85$ & $S, 5.85$ & $(3: 2)$ \\
\hline \multirow{2}{*}{2} & \multirow{2}{*}{$3 b$} & \multirow{2}{*}{57} & \multirow{2}{*}{132} & $48.86^{\circ}$ & $\mathrm{N}, 3.58$ & $\mathrm{~N}, 3.64$ & 0.79 \\
\hline & & & & $(\mathrm{c}, 1.00)$ & S, 5.61 & S, 5.54 & $(3: 2)$ \\
\hline \multirow{2}{*}{3} & \multirow{2}{*}{$3 c$} & \multirow{2}{*}{64} & \multirow{2}{*}{142} & $39.71^{\circ}$ & $\mathrm{N}, 3.60$ & $\mathrm{~N}, 3.64$ & 0.81 \\
\hline & & & & $(\mathrm{c}, 1.03)$ & $S, 5.49$ & $S, 5.54$ & $(3: 2)$ \\
\hline \multirow{2}{*}{4} & \multirow{2}{*}{ 3d } & \multirow{2}{*}{55} & \multirow{2}{*}{135} & $39.73^{\circ}$ & $\mathrm{N}, 3.77$ & $\mathrm{~N}, 3.64$ & 0.83 \\
\hline & & & & $(\mathrm{c}, 1.00)$ & $S, 5.48$ & $S, 5.54$ & $(3: 2)$ \\
\hline \multirow{2}{*}{5} & \multirow{2}{*}{$3 e$} & \multirow{2}{*}{56} & \multirow{2}{*}{152} & $91.22^{\circ}$ & $\mathrm{N}, 3.77$ & $\mathrm{~N}, 3.70$ & 0.66 \\
\hline & & & & $(\mathrm{c}, 0.98)$ & $S, 5.55$ & $S, 5.64$ & $(3: 2)$ \\
\hline \multirow{2}{*}{6} & \multirow{2}{*}{$3 f$} & \multirow{2}{*}{59} & \multirow{2}{*}{129} & $69.54^{\circ}$ & $\mathrm{N}, 3.63$ & $\mathrm{~N}, 3.70$ & 0.79 \\
\hline & & & & $(\mathrm{c}, 1.00)$ & $S, 5.71$ & S, 5.64 & $(3: 2)$ \\
\hline \multirow{2}{*}{7} & \multirow{2}{*}{$3 g$} & \multirow{2}{*}{65} & \multirow{2}{*}{166} & $49.01^{\circ}$ & $\mathrm{N}, 3.63$ & $\mathrm{~N}, 3.70$ & 0.75 \\
\hline & & & & $(\mathrm{c}, 1.02)$ & $S, 5.56$ & S, 5.64 & $(3: 2)$ \\
\hline
\end{tabular}

Satisfactory $C$ and $H$ analysis are found in all cases.

3a: IR (KBr):- v $3468(\mathrm{~N}-\mathrm{H}) ; 2974(\mathrm{Ar}-\mathrm{H}) ; 1730(\mathrm{C}=\mathrm{O}) ; 1602(\mathrm{C}=\mathrm{N}) 1373(\mathrm{C}-\mathrm{N})$; $1271(\mathrm{C}-\mathrm{O}) ; 1095(\mathrm{C}=\mathrm{S}) .{ }^{1} \mathrm{HNMR}\left(\mathrm{CDCl}_{3}\right)$ :- $\delta$ 8.65-7.26 (m, N-H and Ar-H); $5.44-3.97$ (m, glucopyranosyl + galactopyranosyl protons); 2.17-1.08 (m, 4COCH$)$. Mass (FAB):331, 169, 105. 
3c: IR (KBr):- v $3419(\mathrm{~N}-\mathrm{H}) ; 2973(\mathrm{Ar}-\mathrm{H}) ; 1729(\mathrm{C}=\mathrm{O}) ; 1602(\mathrm{C}=\mathrm{N}) 1373(\mathrm{C}-\mathrm{N})$; $1270(\mathrm{C}-\mathrm{O}) ; 1096(\mathrm{C}=\mathrm{S}) .{ }^{1} \mathrm{HNMR}\left(\mathrm{CDCl}_{3}\right): \delta$ 8.3-7.25 (m, N-H and Ar-H); $5.77-3.95(\mathrm{~m}$, glucopyranosyl + galactopyranosyl protons); 2.22-1.06 (m, 4COCH 3 ). Mass (FAB):- 331, $169,105$.

3f: IR (KBr):- v $3451(\mathrm{~N}-\mathrm{H}) ; 2972(\mathrm{Ar}-\mathrm{H}) ; 1730(\mathrm{C}=\mathrm{O}) ; 1602(\mathrm{C}=\mathrm{N}) 1372(\mathrm{C}-\mathrm{N})$; $1271(\mathrm{C}-\mathrm{O}) ; 1094(\mathrm{C}=\mathrm{S}) .{ }^{1} \mathrm{HNMR}\left(\mathrm{CDCl}_{3}\right): \delta$ 8.65-7.26 (m, N-H and Ar-H); 5.44 - $3.97(\mathrm{~m}$, glucopyranosyl + galactopyranosyl protons); 2.17-1.08 (m, 4COCH 3 ). Mass (FAB):- 331, $169,105$.

\section{Antimicrobial activity}

All the compounds were screened for their antibacterial activity against pathogenic bacteria such as E. coli, S. aureus, P. vulgaris and P. aregenosa by cup plate agar diffusion method ${ }^{12,13}$, at a concentration $100 \mu \mathrm{g} / \mathrm{mL}$ in DMSO by using the standard Amikacin at the same concentration. All compounds were also screened for their antifungal activity against $A$. niger and C. albicans by cup plate method at a concentration of $100 \mu \mathrm{g} / \mathrm{mL}$ in DMSO by using the standard Fluconazole at the same concentration. The zone of inhibition was measured in $\mathrm{mm}$ and is average of three readings. The readings are shown in Table 2.

Table 2. Antimicrobial activities of compounds (3a-g).

\begin{tabular}{cccccccc}
\hline Compounds & \multicolumn{4}{c}{ Antibacterial activity } & \multicolumn{2}{c}{ Antifungal activity } \\
\cline { 2 - 7 } & E. coli & S. aureus & P. Vulgaris & P. aregenosa & C. albicans & A. niger \\
\hline 3a & 17 & - & 15 & - & 09 & 14 \\
3b & - & 11 & 13 & - & 08 & 15 \\
3c & 21 & 19 & - & 21 & - & 14 \\
3d & - & 11 & 13 & - & - & - \\
3e & 14 & 17 & 18 & 19 & 11 & 14 \\
3f & - & 11 & - & 15 & - & 19 \\
3g & 09 & 09 & - & 08 & - & - \\
Amikacin & $\mathbf{2 5}$ & $\mathbf{2 3}$ & $\mathbf{2 3}$ & $\mathbf{2 5}$ & - & - \\
Fluconazole & - & - & - & - & $\mathbf{1 5}$ & $\mathbf{2 1}$ \\
\hline
\end{tabular}

Including well diameter of $5 \mathrm{~mm}$.

From the above observations, it is clear that compounds $\mathbf{3 c}$ and $\mathbf{3 e}$ are active against E.coli, S. aureus and P. aregenosa as compared to standard, while $\mathbf{3 b}, \mathbf{3 d}, \mathbf{3 f}$ and $\mathbf{3 g}$ show low or moderate activity against the bacteria.

In antifungal activity, compounds $\mathbf{3 a}, \mathbf{3 b}$ and $\mathbf{3 e}$ show good activity against $C$. albicans while other are inactive and compounds $\mathbf{3 a}, \mathbf{3 b}, \mathbf{3 c}, \mathbf{3 e}$ and $\mathbf{3 f}$ show good activity, while others show low activity against $A$. niger.

\section{Results and Discussion}

Several 2- $S$-tetra- $O$-benzoyl- $D$-glucopyranosyl 1 -aryl-5-tetra- $O$-acetyl- $\beta$ - $D$-galactopyranosyl-2, 4- isodithiobiurets (3a-g) have been synthesized by the interaction of tetra$O$-acetyl- $\beta$ - $D$-galactopyranosyl isothiocyante (1) and various $S$-tetra- $O$-benzoyl- $D$ glucopyranosyl-1-aryl isothiocarbamides (2a-g). All the products were recrystallised form ethanol. The structures of all the synthesized products were developed on the basis of usual chemical transformation and IR, NMR and Mass Spectral analysis ${ }^{6-9}$. The newly synthesized compounds were screened for their antibacterial and antifungal activities. 

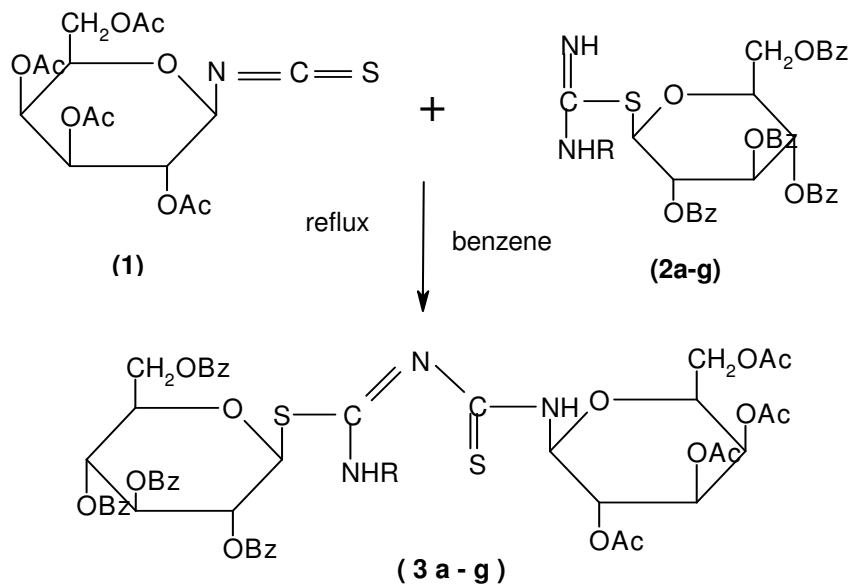

Scheme 1

Where, $R=$ (a) phenyl, (b) o-Cl phenyl, (c) $m-C l$ phenyl, (d) $p-C l$ phenyl, (e) $o-$ tolyl, $(f) m-$ tolyl, $(\mathrm{g}) \mathrm{p}-$ tolyl $, \mathrm{Bz}=\mathrm{COC}_{6} \mathrm{H}_{5}, \mathrm{Ac}=\mathrm{COCH}_{3}$.

\section{Acknowledgment}

Authors are thankful to RSIC, CDRI Lucknow for providing the spectra and also to Dr. S. G. Bhadange, Principal, Shri Shivaji College, Akola for providing necessary facilities.

\section{References}

1 Siddique N and Pandeya S N, Indian J Pharm., 1992, 24, 171-173.

2 Garcia Fernandez J M and Mellet C O, Adv Carbohydr Chem Biochem., 2000, 55, 35-135.

3 Prata C, Mora N, Lacombe J M, Maurizis J C and Pucci B, Carbohydr Res., 1999, 321, 4-14.

4 Todoulou O G, Papadaki-valiraki A E, Filippatos E C, Ikeda S and De Clercq E, Eur J Med Chem., 1994, 29, 127-131.

5 Tripathi R P, Tiwari V K, Tewari N, Katiyar D, Saxena N, Sinha S, Gaikwad A, Srivastava A, Chaturvedi V, Manju Y K, Srivastava R and Srivastava B S, Bioorg Med Chem., 2005, 13, 5668-5679.

6 Isac-Garcia J, Calvo-Flores F G, Hernandez -Mateo F and Santoyo-Gonzalez F, Eur J Org Chem., 2001, 383 - 387.

7 Blanco J L J, Barria C S, Benito J M, Mellet C O, Fuentes J, Santoyo-Gonzalez F and Garcia Fernandez J M, Synthesis, 1999, 11, 1907-1914.

8 Pearson M S M, Robin A, Bourgougnon N, Meslin J C and Denianud D, J Org Chem., 2003, 68, 8583-8587.

9 Saleh M A, Sulfur lett., 2002, 25(6), 235-237.

10 Mahalle P R, Korpe G V and Deshmukh S P, J Indian Chem Soc., 2008, 85, 953-958.

11 Bhagat S K and Deshmukh S P, Asian J Chem., 2002, 14, 243-246.

12 Mangte D V, Deshmukh S P, Bhokare D D, Deshpande A R, Indian J Pharm Sci., 2007, 69(2), 295-298.

13 Matsumura S, Imai K, Voshikawa S, Kawada K and Uchibari T, JAOCS, 1990, 67(12), 996-1001. 


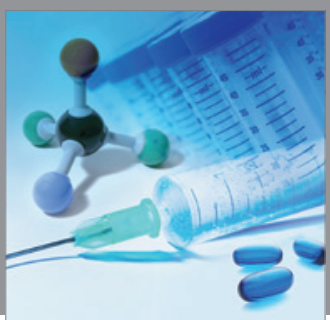

International Journal of

Medicinal Chemistry

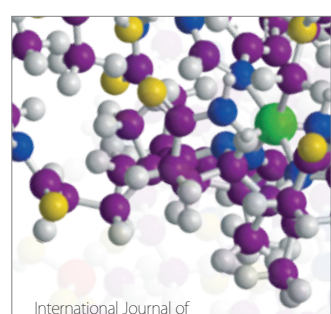

Carbohydrate Chemistry

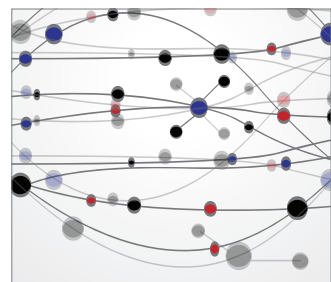

The Scientific World Journal
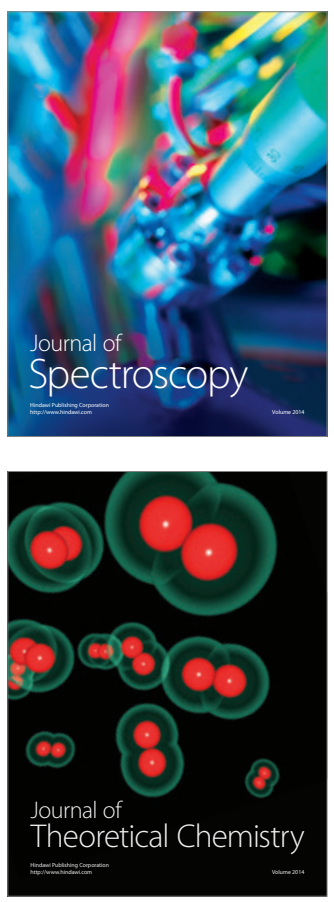
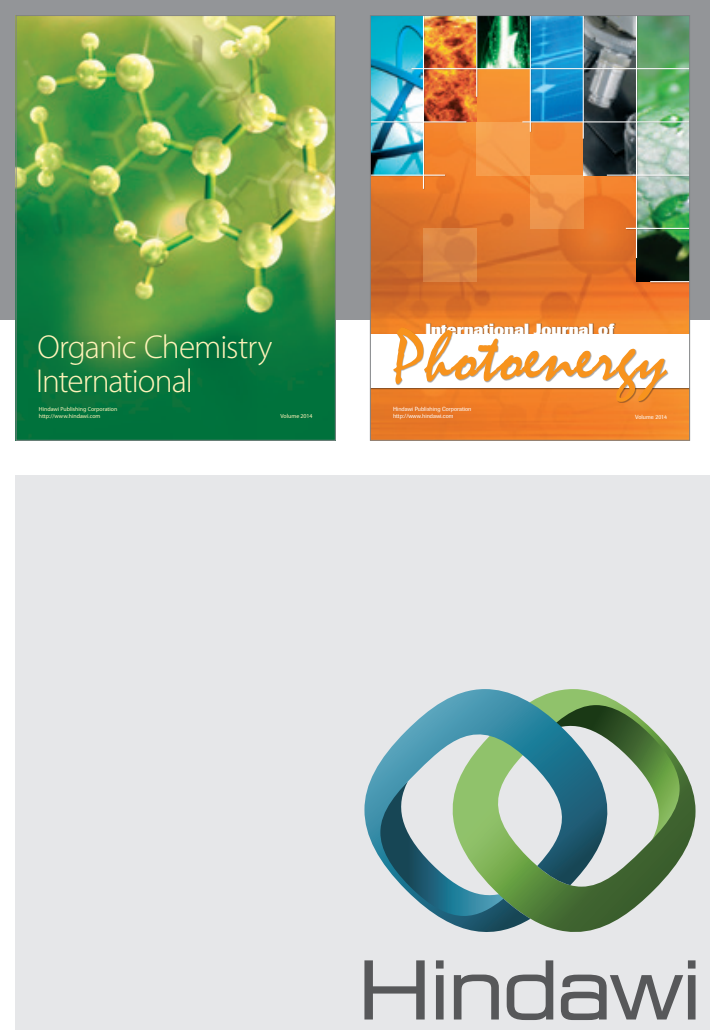

Submit your manuscripts at

http://www.hindawi.com
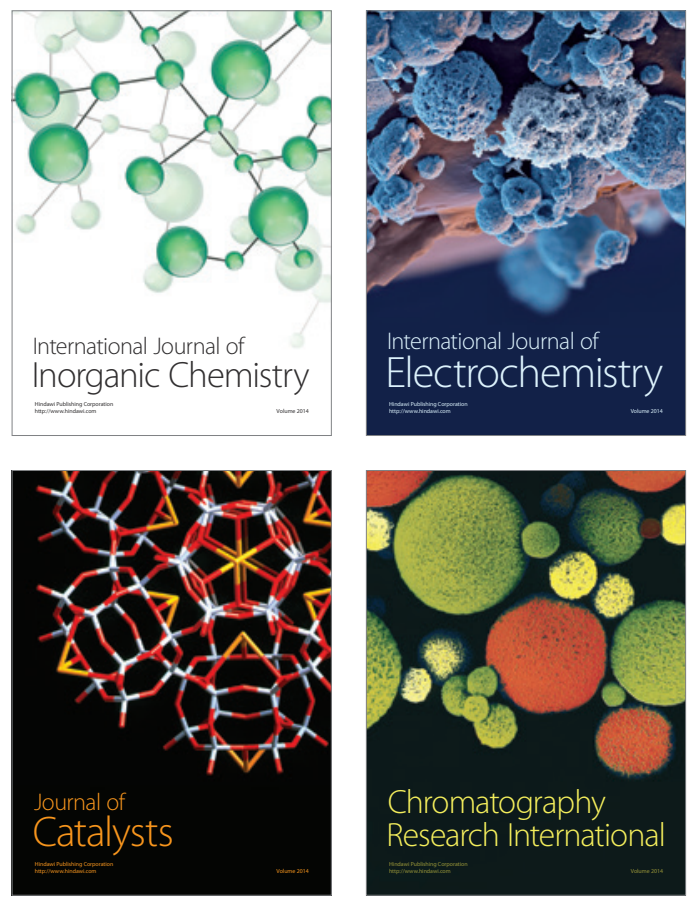
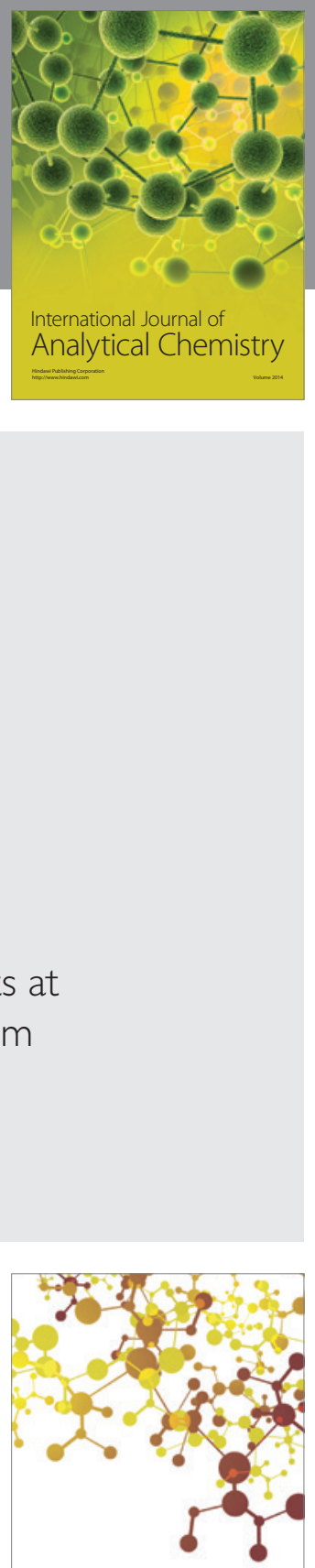

Journal of

Applied Chemistry
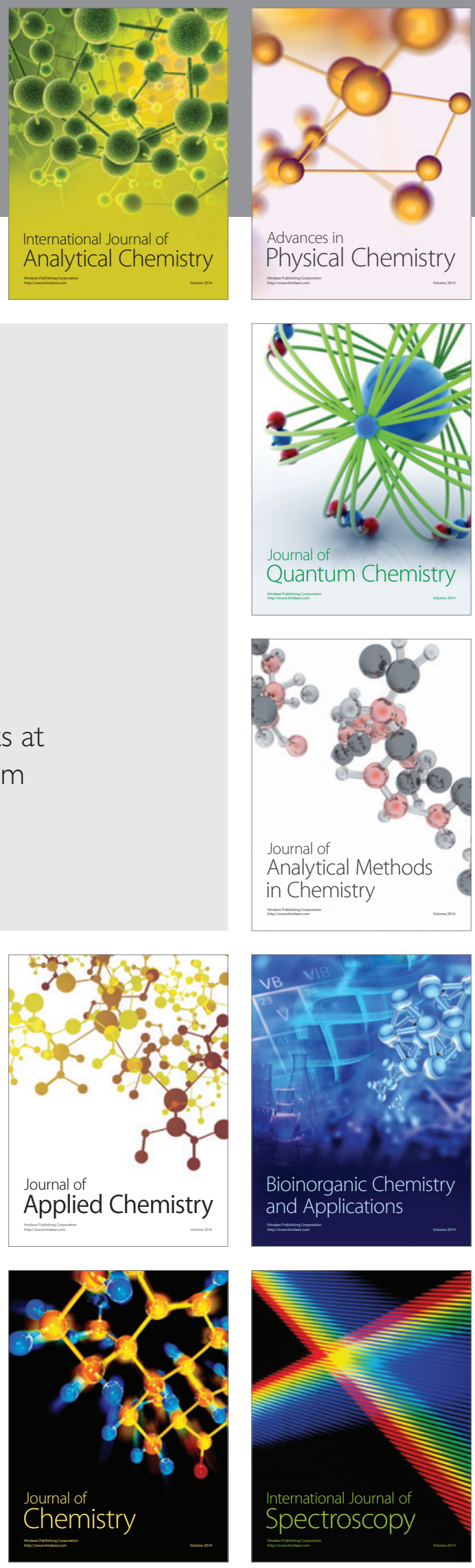